\title{
AVALIAÇÃO DA INFLUÊNCIA DE 2 DIFERENTES VEÍCULOS BAJA NA ATIVIDADE MUSCULAR E MOVIMENTOS DO PILOTO: UM ESTUDO DE CASO
}

Guilherme da Silva Bertolaccini (1);

Sara R. Martins da Silva (2);

Josieli Aparecida Marques Boiani (3);

Lais Faganello (4);

Rafael Kenji Nakajima (5);

Idinei Francisco de Carvalho Filho (6);

Ana Carolina Bianchi (7);

Fausto Orsi Medola (8);

Luis Carlos Paschoarelli (9).

(1) PPGDesign-UNESP, Mestrando em Design

guilhermebertolaccini@gmail.com

(2) PPGDesign-UNESP, Mestranda em Design

e-mail: s.martinsto@gmail.com

(3) PPGDesign-UNESP, Mestranda em Design

e-mail: jboiani@yahoo.com.br

(4) PPGDesign-UNESP, Mestre em Design

e-mail: laisfaganello@hotmail.com

(5) PPGDesign-UNESP, Graduando em Design

e-mail: rafa.nakajima@gmail.com

(6) PPGDesign-UNESP, Graduando em Design

e-mail: idinei.francisco@gmail.com

(7) PPGDesign-UNESP, Graduanda em Design

e-mail: acbianchi1802@gmail.com.br

(8) PPGDesign-UNESP, Professor Assistente Doutor

e-mail: fausto.medola@faac.unesp.br

(9) PPGDesign-UNESP, Professor Associado Doutor

e-mail: paschoarelli@faac.unesp.br 


\section{RESUMO}

Este estudo objetivou avaliar a influência de dois diferentes modelos de veículo BAJA na atividade muscular e movimentos dos membros superiores e inferiores durante a condução simulada de prova off-road. De caráter preliminar este trabalho faz parte de uma maior avaliação ergonômica que visa fornecer dados que fundamentam a elaboração e adequações de novos projetos de veículos Baja. Neste sentido, a avaliação da postura e movimentos realizados pelo piloto na condução do veículo poderá apontar condições de possível sobrecarga biomecânica, as quais podem fundamentar ajustes posteriores no projeto do veículo.

\section{ABSTRACT}

This study aimed to evaluate the influence of two different BAJA vehicle models in muscle activity and movements of upper and lower limbs during simulated driving off-road race. In this preliminary work is part of a larger ergonomic evaluation that aims to provide data that underlie the preparation and adjustments to new Baja vehicle projects. In this sense, the body posture and movements performed by the driver in driving the vehicle can point conditions can biomechanical overload, which can support subsequent adjustments in vehicle design.

\section{INTRODUÇÃO}

Segundo o Anuário da Indústria Automobilística Brasileira - ANFAVEA (2016), o Brasil é o $8^{\circ}$ produtor mundial de autoveículos com 65 fábricas em 11 estados e capacidade de produção de 4,5 milhões de unidades, o que gera um faturamento de US $\$ 95,5$ bilhões (incluindo autopeças) e emprega 1,5 milhão de pessoas. O setor também investe consideravelmente em pesquisa e desenvolvimento, sendo responsável por várias inovações, dentre as quais destaca-se o carro movido a etanol, que se tornou um combustível renovável de sucesso em todo o mundo (ANFAVEA, 2016).

Inicialmente, o ato de utilizar o automóvel era disposto como substituto aos veículos de tração animal, sendo um ícone do século $X X$, estando relacionado à um estilo de vida moderno e passando a ser encarado como uma atividade esportiva (MELO, 2008). Dentro do âmbito das competições existem diversas modalidades como Formula 1, Formula E, Nascar, Dakar Rally, 24 Horas de Le Mans, entre outros. Também podemos destacar as competições de aperfeiçoamento automobilísticos, que ocorrem dentro das universidades como o Fórmula SAE e o BAJA SAE.

A competição BAJA SAE surgiu em 1976 na Universidade da Carolina do Sul (EUA), com o objetivo de projetar e fabricar um carro todo terreno (off-road) com mais de duas rodas, para apenas um ocupante (Figura 1), que fosse o veículo mais competitivo possível pelo menor custo (SAE, 2016).

Expandindo-se pelo mundo, a modalidade chegou ao Brasil em 1991, tendo a primeira competição realizada em 1995 (BRASIL, 2016). Atualmente, o Baja SAE possui competições no mundo todo, com etapa mundial no Canadá a cada ano, na qual participam os três primeiros colocados de cada país. O BAJA SAE Brasil integra várias universidades de todo o país e transformou-se em um exemplo de sucesso na qualificação dos futuros profissionais automobilísticos (IKEDA, 2011). A modalidade foi criada pela Sociedade de Engenheiros da Mobilidade (SAE Brasil), como forma de incentivar e fortalecer os estudos em desenvolvimento de automóveis no país, sendo 
hoje um dos maiores programas para capacitar futuros profissionais para a indústria automobilística, (SANTOS, 2015).

Figura 1: Baja Modelo 1976.

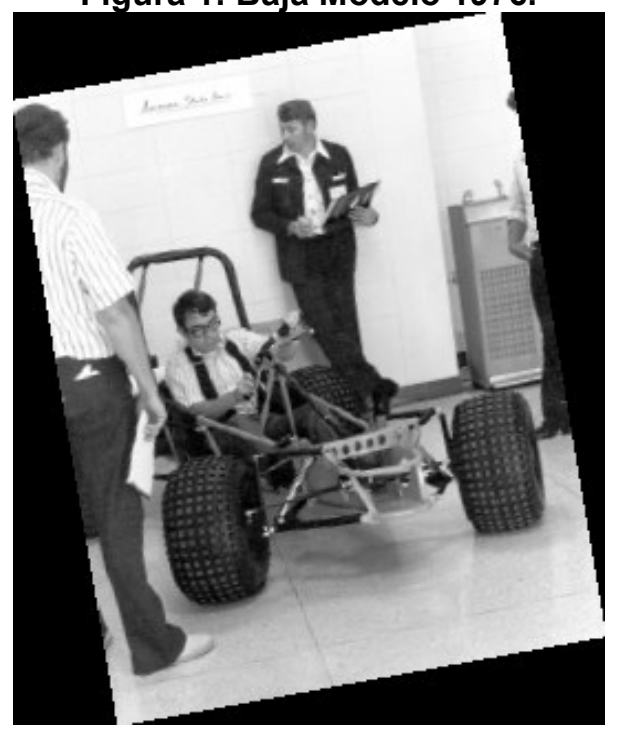

Fonte: www.sae.org/students.

A disputa consiste em diversas provas estáticas e dinâmicas que objetivam avaliar as competências de cada projeto. São provas que avaliam aspectos técnicos, funcionais, ergonômicos e até mesmo estéticos. A disputa possibilita aos participantes aplicar os conhecimentos teóricos na prática, construindo um veículo off-road que suporte condições de um terreno acidentado. Dentre as etapas de avaliação encontra-se o enduro, que envolve o piloto em situações de resistência física, o qual permanece por cerca de quatro horas dirigindo o veículo.

De acordo com o estudo de Ikeda et al. (2011), as condições ergonômicas para o condutor de um veículo, quando inadequadas, podem influenciar seu desempenho devido aos níveis elevados de desconforto corporal. Ao dirigir um veículo BAJA, o condutor executa de forma repetitiva movimentos de pequena amplitude que exigem esforço devido à mecânica do veículo e a precisão requerida da atividade. Neste sentido, o espaço interno do veículo representa um importante fator ergonômico. Segundo Santos (2015), este deve ser de fácil acesso aos mecanismos e permitir movimentos das mãos e dos pés no momento de acioná-los.

Por se tratar de uma prova de longa duração (4 horas), o conforto postural desempenha um papel primordial para o bom desempenho do piloto durante a prova. Neste sentido, a avaliação da postura e movimentos realizados pelo piloto na condução do veículo poderá apontar condições de possível sobrecarga biomecânica, as quais podem fundamentar ajustes posteriores no projeto do veículo.

O presente estudo teve como objetivo avaliar a influência de dois diferentes modelos de veículo BAJA na atividade muscular e movimentos dos membros superiores e inferiores durante a condução em simulação de prova off-road. De caráter preliminar, este estudo é parte de uma ampla abordagem de avaliação ergonômica que foi 
realizada com o objetivo de fornecer dados que fundamentam adequações no projeto do veículo.

\section{MATERIAIS E MÉTODOS}

Esta pesquisa caracteriza-se como um estudo de caso envolvendo um piloto da equipe Pac Baja da UNESP-Bauru, do gênero masculino, destro, com altura de $1,75 \mathrm{~m}$ e $65 \mathrm{~kg}$. Foram avaliados dois modelos de veículo BAJA, com características distintas de posicionamento e suporte postural do piloto (Figuras 2 e 3 ).

Figura 2: Modelo 2015 e Modelo 2013.

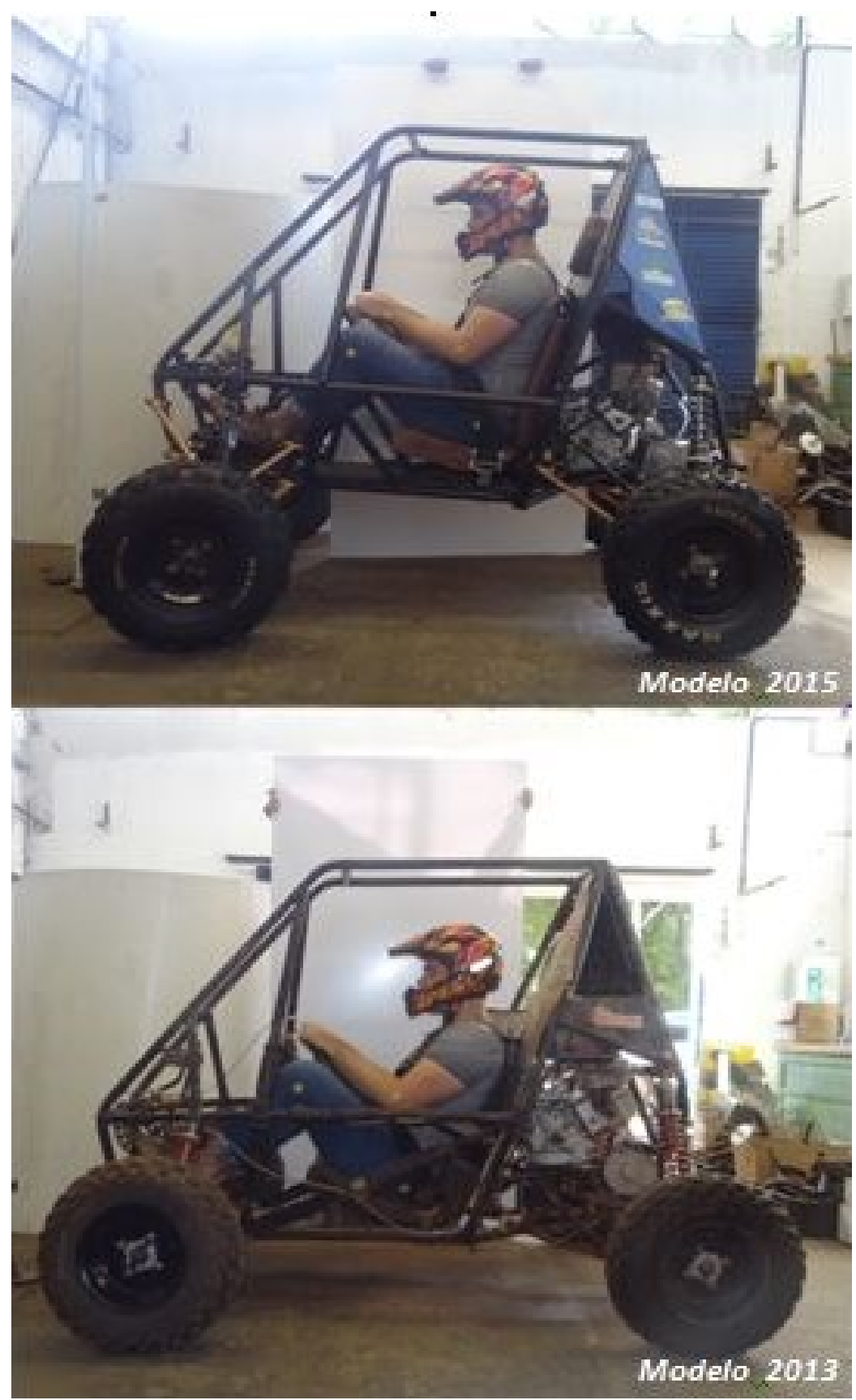

Fonte: Elaborado pelos autores. 
Figura 3: Dimensões Modelo 2015 e Modelo 2013
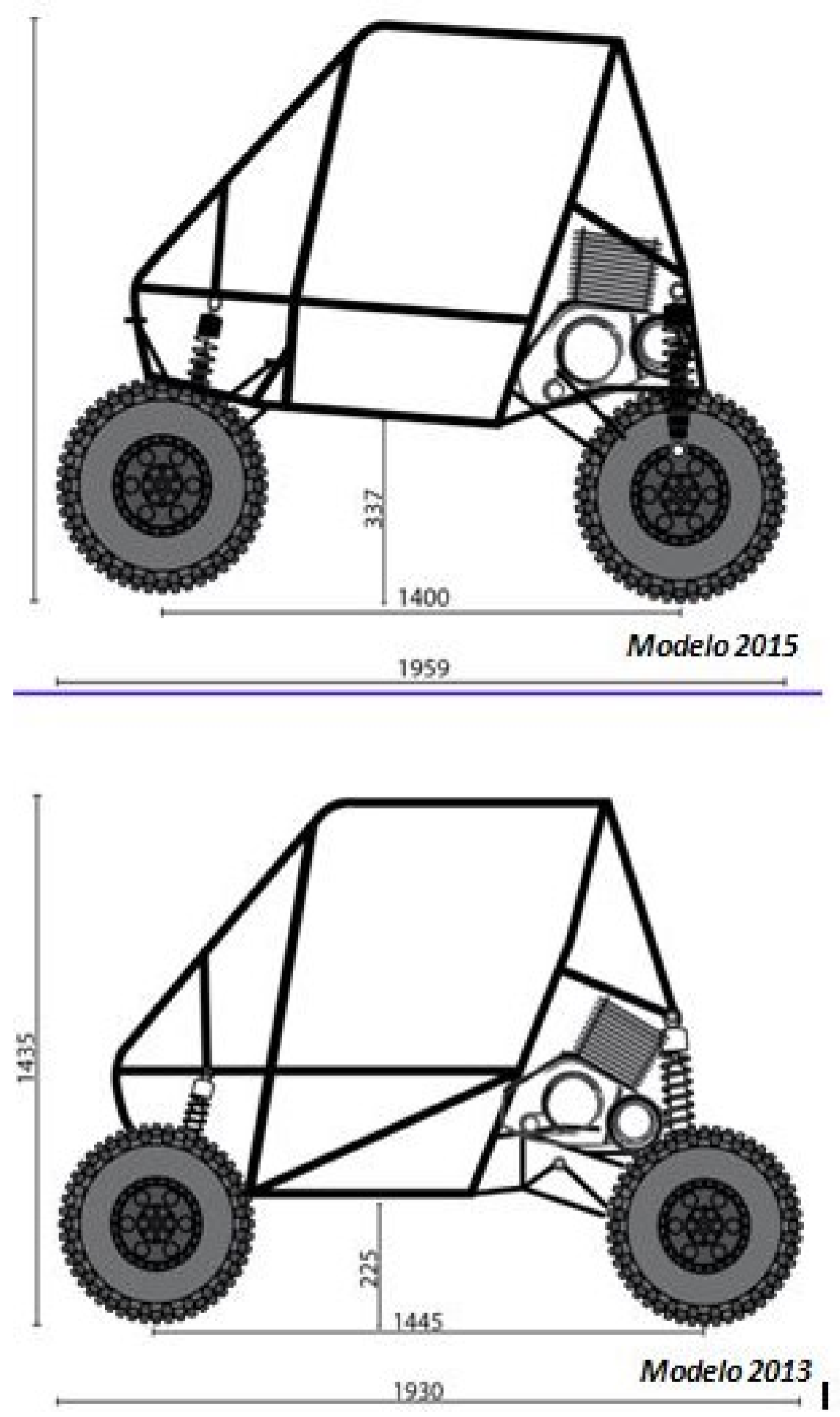

Fonte: Elaborado pelos autores

O teste consistiu na condução do veículo por um trajeto off-road, (Figura 2) de (1,6 $\mathrm{Km}$ ), dentro do Campus da UNESP-Bauru, com características similares às encontradas durante uma corrida oficial da competição campeonato BAJA-SAE. O 
piloto foi solicitado a dirigir o veículo em condições similares a uma competição. Antes do teste, o piloto conduziu o carro pelo percurso para conhecer as condições de terreno e trajetória.

Figura 4: Mapa do trajeto

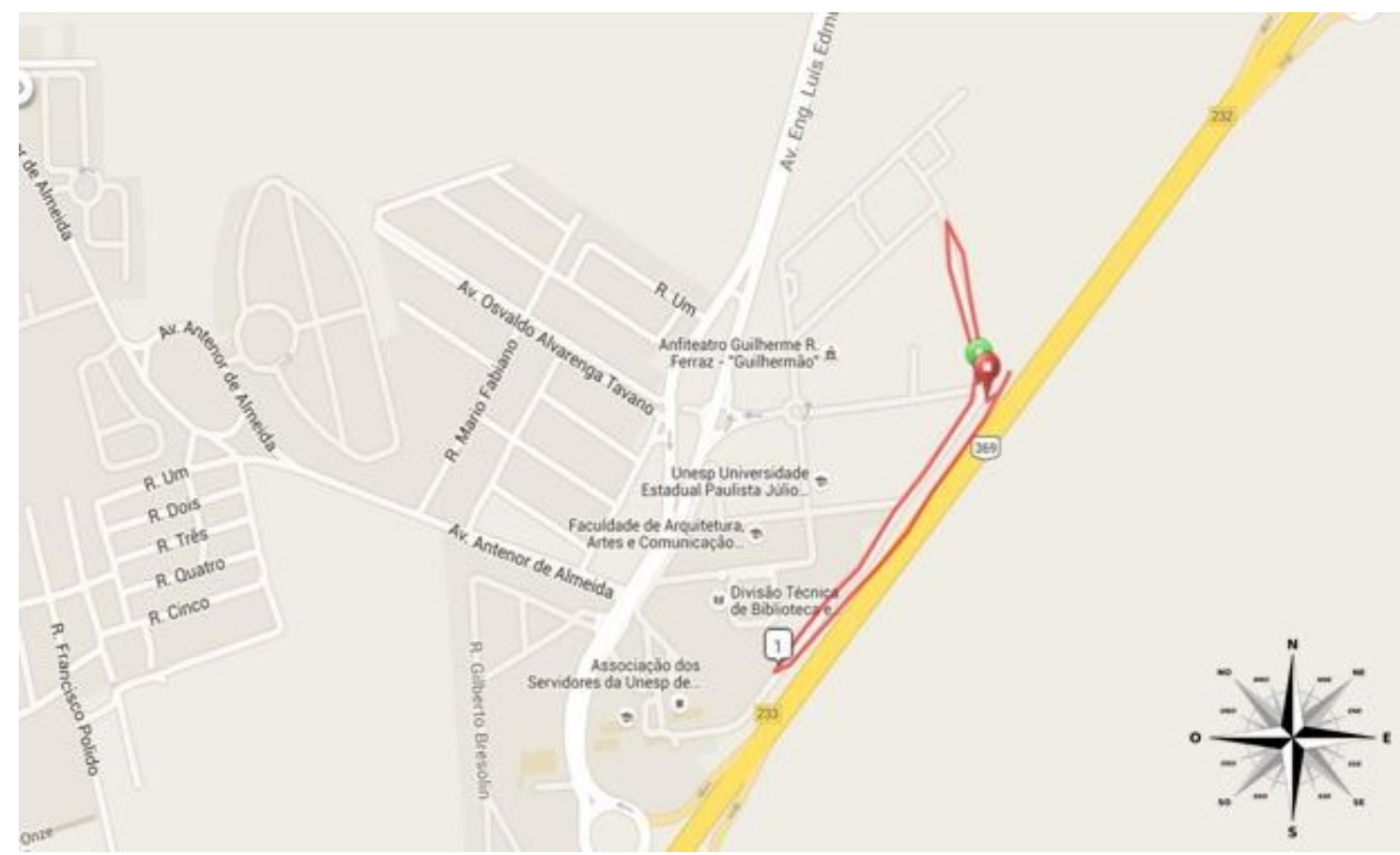

Fonte: Elaborado pelos autores

Durante o teste, foram coletados dados da atividade elétrica dos músculos deltoide anterior, flexores dos dedos, tibial anterior e gastrocnêmio lateral, e dados dos ângulos articulares de ombro, cotovelo e joelho. Para isto, foram utilizados sensores wireless T-sens sEMG e T-sens Motion CAPTIV (TEA Ergo, Nance, França) e eletrodos de superfície Triodo modelo T3402M (Thought Technology, Montreal, Canadá), todos colocados no lado dominante (direito). O registro do sinal de eletromiografia foi feito a $2048 \mathrm{~Hz}$ com $128 \mathrm{~Hz}$ de cálculo de filtragem RMS e analisados com o software CAPTIV L-7000 (TEA Ergo, Nance, França).

A colocação do eletrodo no deltoide anterior deu-se a um dedo de largura distal e anterior ao acrômio. Para o registro da atividade dos flexores dos dedos o eletrodo foi colocado no ventre muscular do antebraço. Já no tibial anterior o eletrodo foi colocado a $1 / 3$ da linha entre a ponta da fíbula e a ponta do maléolo medial. $E$ para o gastrocnêmio lateral o eletrodo foi colocado a $1 / 3$ da linha entre a cabeça da fíbula e 0 calcanhar. Estes procedimentos foram realizados seguindo as instruções do SENIAM Surface Electromiography of Non-Invasive Assessment of Muscles (seniam.org) e através da anatomia palpatória.

Os sensores de movimento foram colocados no braço, antebraço, coxa e perna do lado direito do piloto. Os ângulos de extensão e flexão do cotovelo, ombro e joelho foram avaliados, juntamente com os ângulos de adução e abdução do joelho e ombro. 
A análise dos dados de EMG e movimento articular foi realizada no software CAPTIV L-7000 (TEA Ergo, Nance, França).

Os aspectos éticos foram respeitados através da aplicação do Termo de Consentimento Livre e Esclarecido (TCLE), que atende a Resolução 466/12-CNS-MS e o "Código de Deontologia do Ergonomista Certificado - Norma ERG BR 1002 ABERGO” (ABERGO, 2003).

Por se tratar de um estudo preliminar que envolveu a participação de apenas um sujeito, os dados serão apresentados de forma descritiva (média e desvio padrão), e não será realizada análise estatística para comparação das médias entre os dois veículos.

\section{RESULTADOS E DISCUSSÕES}

\subsection{Eletromiografia (EMG)}

Os dados de eletromiografia apontam discretas diferenças na atividade elétrica muscular entre os dois modelos de veículos BAJA, e são apresentados na Figura 6.

Figura 5: Média e desvio padrão de EMG dos músculos de membros superiores e inferiores.

\section{Eletromiografia}

Modelo 2013 Modelo 2015

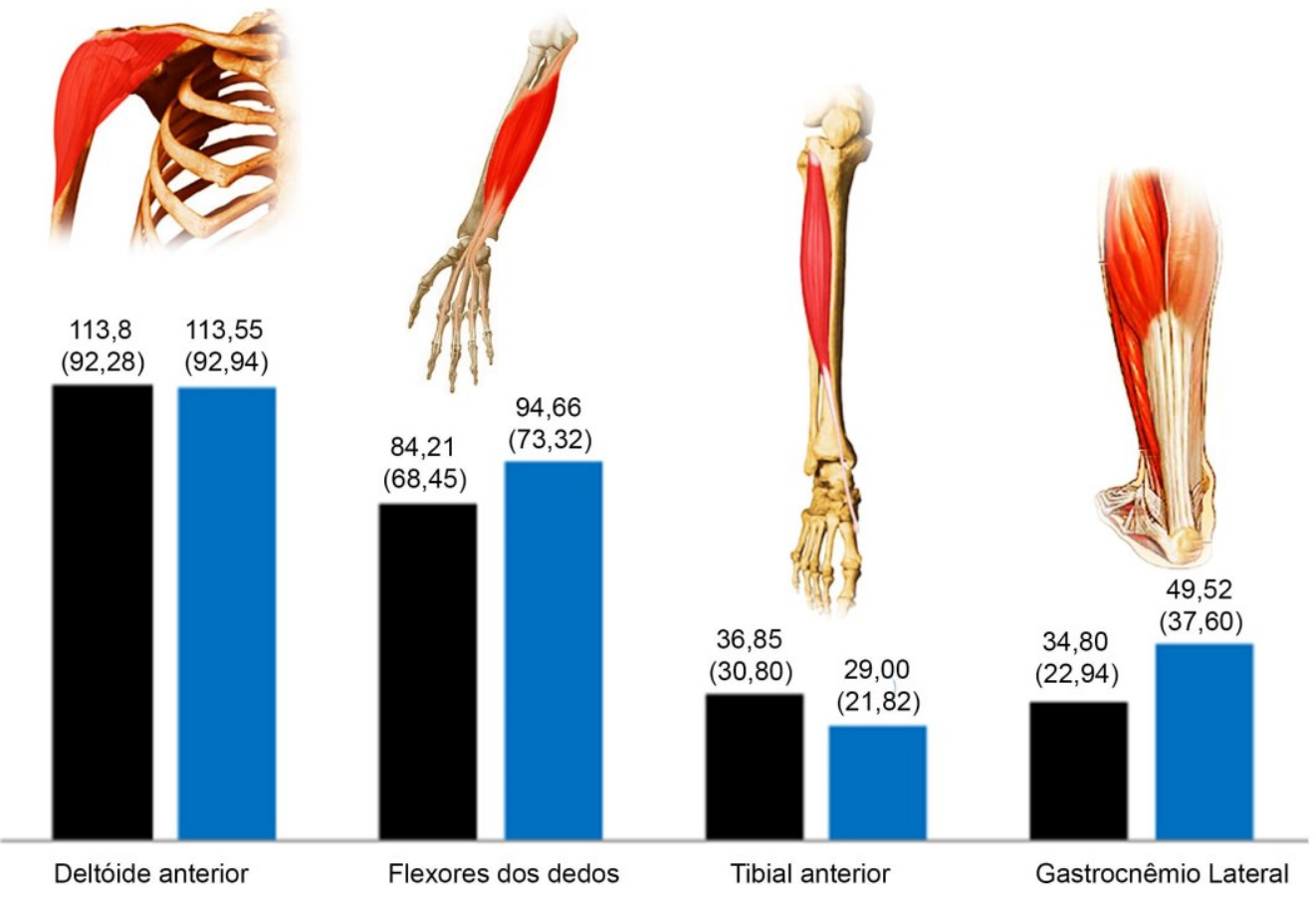

Fonte: Elaborado pelos autores. 
Os dados de ambos os veículos demonstram, de forma preliminar, que o deltoide anterior teve ativação muscular similar durante o uso de ambos os veículos, sendo também o músculo mais solicitado durante o percurso em ambos os carros $(113,8 \mathrm{mV}$ para o modelo 2013 e 113,55 para o modelo 2015). Enquanto os músculos flexores dos dedos apresentaram uma maior ativação no modelo 2015 (94,66 mV). Evidenciam também a relação de antagonismo entre os músculos tibial anterior e gastrocnêmio lateral, principalmente no modelo 2015 , o qual os valores de ativação no gastrocnêmio $(49,52 \mathrm{mV})$ foram maiores que no tibial $(29 \mathrm{mV})$, já o modelo 2013 demonstra uma relação mais equilibrada entre os 2 músculos $(36,85 \mathrm{mV})$ para o tibial e $(34,8 \mathrm{mV})$ para o gastrocnêmio.

\section{2 Ângulos articulares}

Os dados aqui apresentados referem-se a uma situação dinâmica, na qual os ângulos articulares do sujeito foram analisados durante a realização da tarefa. Os ângulos mínimos, máximos e a amplitude articular (delta) de flexão/extensão e adução/abdução do ombro, flexão/extensão de cotovelo e flexão/extensão de joelho são apresentados na Tabela 1.

Tabela 1: Ângulo articulares de ombro, cotovelo e joelho. Os valores mínimos referem-se a flexão e adução, e os valores máximos extensão e abdução.

\begin{tabular}{|c|c|c|c|c|c|c|}
\hline & & \multicolumn{2}{|c|}{ OMBRO } & \multirow{2}{*}{$\begin{array}{c}\text { COTOVELO } \\
\text { Fle./Ext. }\end{array}$} & \multicolumn{2}{|c|}{ JOELHO } \\
\hline \multirow{4}{*}{ Modelo 2013} & & Ext./Flex. & Abd./Add. & & Flex./Ext. & Abd./Add. \\
\hline & Max. & $78,09^{\circ}$ & $80,75^{\circ}$ & $146,46^{\circ}$ & $90,09^{\circ}$ & $-4,41^{\circ}$ \\
\hline & Min. & $-0,67^{\circ}$ & $15,03^{\circ}$ & $90,14^{\circ}$ & $53,68^{\circ}$ & $-49,32^{\circ}$ \\
\hline & Delta & $77,42^{\circ}$ & $65,72^{\circ}$ & $56,32^{\circ}$ & $36,41^{\circ}$ & $44,91^{\circ}$ \\
\hline \multirow{3}{*}{ Modelo 2015} & Max. & $71,8^{\circ}$ & $79,97^{\circ}$ & $112,4^{\circ}$ & $58,66^{\circ}$ & $-5,25^{\circ}$ \\
\hline & Min. & $0,73^{\circ}$ & $-16,38^{\circ}$ & $42,49^{\circ}$ & $37,85^{\circ}$ & $-31,89^{\circ}$ \\
\hline & Delta & $71,07^{\circ}$ & $96,35^{\circ}$ & $69,91^{\circ}$ & $20,81^{\circ}$ & $26,64^{\circ}$ \\
\hline
\end{tabular}

Fonte: Elaborado pelos autores

Os resultados demonstram que o piloto realizou a atividade de condução do veículo em uma postura de maior flexão no modelo 2015, uma vez que os ângulos mínimos e máximos referentes à flexão de cotovelo $\left(42,49^{\circ}\right.$ e 112,4$)$ e joelho $\left(37,85^{\circ}\right.$ e $\left.58,66^{\circ}\right)$ estão em uma amplitude articular que se inicia em posição de maior flexão em

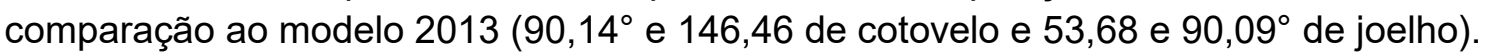
Além disso, o movimento de flexão de ombro foi discretamente maior no veículo modelo $2013\left(78,09^{\circ}\right)$ em comparação ao modelo $2015\left(71,8^{\circ}\right)$, o que reforça o argumento de que o veículo 2015 induz o piloto a uma postura mais flexionada, o que pode ser consequência da menor distância entre-eixos neste modelo. 
O presente estudo apresenta limitações que precisam ser consideradas na interpretação dos resultados. Primeiro, por ter sido avaliado apenas um sujeito, os resultados podem não ser representativos de uma amostra mais numerosa de pilotos BAJA. Além disso, o tempo de análise da coleta foi de aproximadamente dois minutos, enquanto uma prova real dura aproximadamente quatro horas. No levantamento bibliográfico realizado, não foram encontrados estudos de parâmetros ergonômicos para veículos BAJA, o que dificulta a discussão dos achados com a literatura.

\section{CONCLUSÕES}

O presente estudo faz parte de uma avaliação, na qual diversos aspectos de dois diferentes veículos Baja da equipe Pac Baja da UNESP-Bauru foram analisados. Buscou-se compreender os aspectos biomecânicos, atividade muscular e movimentos, de pilotos de veículos Baja por meio de comparação de dois diferentes projetos de veículo.

Diversos são os estudos que abordam as questões ergonômicas nos mais diferentes tipos de veículos, entretanto não foram encontrados estudos que realizaram uma abordagem destes parâmetros com veículos Baja, o que dificulta a discussão com a literatura. Porém os dados, aqui apresentados, apesar de preliminares, são de extrema importância para equipes de veículos Baja aperfeiçoarem seus projetos, uma vez que uma abordagem dessa natureza pôde gerar uma comparação entre dois diferentes projetos.

\section{AGRADECIMENTOS}

O presente estudo teve apoio das agências financiadoras CAPES e CNPq (proc. 458740/2013-6).

\section{REFERÊNCIAS}

ABERGO. Norma ERG BR 1002 - Código de Deontologia do Ergonomista Certificado. Associação Brasileira de Ergonomia, 2003

ANFAVEA. Associação Nacional dos Fabricantes de Veículos Automotores. Anuário da Indústria Automobilística Brasileira - 2016. Gráfica Mundo. São Paulo, 2016.

BRASIL,B. S. .BAJA. Disponível em:<http://portal.saebrasil.org.br/programas-estudantis/bajasae-brasil>. Acesso em: 25 de jun. 2016.

IKEDA, A. Y., PASCHOARELLI, L. C., DEGANUTTI, R. Desconforto percebido em condutores de veículos Baja - Parâmetros para o design ergonômico do assento. VI Congresso Internacional de Pesquisa em Design - CIPED. Lisboa, 2011.

MELO, V. A. de. O automóvel, o automobilismo e a modernidade no Brasil (1891-1908). Rev. Bras. Cienc. Esporte, Campinas, v. 30. n. 1, p. 187-203, set 2008. 
SAE, International. Disponível em: <http://www.sae.org/student> Acesso em 16 de jul.

SANTOS, A. B. dos. Estudo ergonômico do veículo Baja do Centro Univeritário

UNIVATES. Monografia. Engenharia de Produção - Centro Universitário UNIVATES, Lajeado, 03 dez. 2015. 65 p. Disponível em: <http://hdl.handle.net/10737/1000>. 